\title{
Análisis genético molecular en Atrofia Muscular Espinal
}

\author{
CARLOS I. VIÑAS P. ${ }^{1}$, IVONNE MARTÍN H. ${ }^{2}$, TATIANA ZALDIVAR V. ${ }^{3}$, \\ NICOLÁS GARÓFALO G. ${ }^{4}$, MARIESKY ZAYAS G. ${ }^{5}$, ROSA GUERRA B. ${ }^{6}$, \\ LUCÍA MARGARITA N. ${ }^{7}$, JOSÉ VARGAS D. ${ }^{8}$, JOEL GUTIÉRREZ G. ${ }^{9}$
}

1. Máster en Genética Médica. Profesor Auxiliar Genética y Biología Molecular. Departamento de Neurogenética. Instituto de Neurología y Neurocirugía (INN), Cuba.

2. Máster en Bioética. Profesor Auxiliar Bioética y Biología Molecular. Departamento de Neurogenética. INN, Cuba.

3. Especialista II grado Genética Clínica. Profesora Auxiliar. Departamento de Neurogenética. INN, Cuba.

4. Especialista II grado Neurología. Profesor Auxiliar Neurología. INN, Cuba.

5. Lic. Tecnología de la Salud. Técnico de laboratorio. INN, Cuba.

6. Técnico de Laboratorio. INN, Cuba.

7. Especialista I grado Pediatría. INN, Cuba.

8. Profesor Titular y Consultante Pediatría. Especialista II grado Pediatría. INN, Cuba.

9. Especialista II grado Neurofisiología. Profesor Titular. INN, Cuba.

\begin{abstract}
Molecular evaluation in infantile spinal muscular atrophy

Introduction: Infantile Spinal Muscular Atrophy (infantile SMA) is a neurodegenerative disease caused primarily by the deletion of the SMN1 gene at 5q11.1 - 13.3. Its severity ranges from type I, in early childhood, to type IV. Objectives: To describe molecular findings in patients with SMA, who were nationally referred to the Institute of Neurology and Neurosurgery and to describe the frequency per 100,000 people in each province of the island. Patients and Methods: 105 patients, referred between 1997 and 2011, were studied. The polymerase chain reaction (PCR) technique was used to detect the deletions of exons 7 and 8 of the SMN1 gene, and DraI and DdeI enzymes, in 2\% agarose gel, were used for digestion. Results: $59 \%$ of the patients were diagnosed with SMA I, $28.6 \%$ with SMA II and $12.4 \%$ with SMA III. $36.2 \%$ of total patients presented deletions of exons $7,8,35.2 \%$ only deletion of exon 7 and $28.2 \%$ did not present deletion of exons. Conclusión: These results are discussed according to the international literature. The frequency per 100,000 inhabitants in each province is presented and discussed according to ancestral diversity of the Cuban population. Also, the originality of the study is mentioned as it is the first report of this type in a Caribbean population.

(Key words: Spinal muscular atrophy, molecular biology, neurodegenerative diseases, SMN gene, Cuba). Rev Chil Pediatr 2013; 84 (5): 499-504
\end{abstract}

Recibido el 13 de junio de 2012, devuelto para corregir el 29 de agosto de 2012, segunda versión 9 de septiembre de 2012, tercera versión 22 de enero de 2013, cuarta versión 16 de septiembre de 2013, aceptado para publicación el 23 de septiembre de 2013.

Este trabajo cumple con los requisitos sobre consentimiento /asentimiento informado, comité de ética, financiamiento, estudios animales y sobre la ausencia de conflictos de intereses según corresponda.

Correspondencia a:

Ivonne Martín H.

E-mail: ivonne.martin@infomed.sld.cu 


\section{RESUMEN}

Introducción: La atrofia muscular espinal de la infancia (AMEi) es una enfermedad neurodegenerativa, causada principalmente por deleciones del gen SMN 1 en su locus 5q11.1-13.3. La severidad va desde el tipo I, que compromete la vida en edades tempranas, hasta el tipo IV. Objetivos: Se describen hallazgos moleculares en pacientes con AME, nacionalmente remitidos al Instituto de Neurología y Neurocirugía así como se reporta la frecuencia por 100.000 habitantes en cada una de las provincias de la isla. Pacientes y Métodos: 105 pacientes fueron estudiados, remitidos entre 1997 y 2011. Para la detección de las deleciones se utilizó la técnica de reacción en cadena de la polimerasa (PCR) con cebadores correspondientes a los exones 7 y 8 del gen SMN 1, y digestión con enzimas DraI y DdeI respectivamente y analizados en gel de agarosa al $2 \%$. Resultados y Discusión: Se encontró un 59\% diagnosticados como AME I, 28,6\% AME II, 12,4\% AME III. Del total de casos 36,2\% resultaron tener deleción de los exones 7 y $8,35,2 \%$ deleción del exón 7 solamente y $28,2 \%$ no presentaron deleción de los exones correspondientes. Se discuten dichos resultados de acuerdo a la literatura internacional. Se presentan los resultados de frecuencias por 100.000 habitantes en cada provincia del país y discuten dichas frecuencias de acuerdo a la diversidad ancestral de la población cubana. Se concluye lo novedoso del estudio que constituye el primer reporte en una población caribeña.

(Palabras clave: Atrofia muscular espinal, biología molecular, enfermedades neurodegenerativas, gen SMN, Cuba).

Rev Chil Pediatr 2013; 84 (5): 499-504

\section{Introducción}

La atrofia muscular espinal (AME) comprende un grupo de enfermedades de causa genética caracterizadas por degeneración de las motoneuronas del asta anterior de la médula espinal y del tallo cerebral. Estas enfermedades presentan una herencia autosómica recesiva y están causadas por deleciones homocigóticas o mutaciones en el gen SMN1 en el locus 5q11.2-13.3. El espectro de la AME comprende desde la afectación severa en el tipo I o Enfermedad de Werdnig Hoffmann a la relativamente benigna tipo III o Enfermedad de Kugelberg-Welander. La determinación de las deleciones de al menos el exón 7 del gen SMN constituye una herramienta para el diagnóstico de esta enfermedad ${ }^{1-3}$.

Se plantea que constituyen la causa genética más frecuente de muerte en la infancia de causa genética después de la fibrosis quística, sin que hasta la fecha se conozca un tratamiento curativo efectivo a pesar del desarrollo alcanzado en la comprensión etiológica de las mismas.

Existen reportes en la literatura donde se plantean cifras de incidencia en diferentes poblaciones, tales como Inglaterra, donde Pearn reportó una incidencia de 4 por 100.000 nacidos vivos, la que sugiere una frecuencia de portadores de 1 en 80 . En Italia la incidencia fue de 7,8 por 100.000 nacidos vivos, para una frecuencia de portadores de 1 en 57 . En Alemania en 1992 un estudio reporta una incidencia de 10 por 100,000 nacidos vivos, lo que sugiere una frecuencia de portadores de 1 en $57^{4-6}$.

En Cuba se plantea una incidencia para la AME I de 3,57 por 100.000 nacidos vivos, determinada por Zaldívar et al, en el 2005, que reportan incluso frecuencias mucho menores en cubanos con ancestros africanos y confirmación por estudios moleculares ${ }^{7}$.

El objetivo de este trabajo es realizar una caracterización molecular de pacientes con impresión diagnóstica de AME, remitidos al Departamento de Neurogenética del Instituto de Neurología y Neurocirugía (INN) en el período 1997-2011, así como reportar los valores de frecuencia por 100.000 habitantes para cada provincia del país.

\section{Pacientes y Método}

Se estudiaron 105 pacientes con sospecha clínica y/o impresión diagnóstica de AME re- 
mitidos al servicio de Neurogenética del Instituto de Neurología y Neurocirugía entre 1997 y 2011.

Se evaluó un $94 \%$ de los casos por Neurólogos, Neonatólogos, Pediatras y/o Genetistas clínicos de los diferentes servicios de Neurología, Neonatología y Pediatría existentes en el país y de la Red Nacional de Genética, los cuales fueron remitidos al INN para ser seguidos por un equipo multidisciplinario constituido por: genetistas, neuropediatras, neurofisiólogos y biólogos moleculares.

\section{Método}

Se registraron los datos mediante la revisión de las historias clínicas. Se registraron la edad de debut de los primeros síntomas, edad en el momento de realizar el diagnóstico, el sexo, el color de la piel, estudios complementarios realizados y los elementos clínicos presentes a la edad del diagnóstico: presencia de debilidad muscular y distribución de la misma, presencia de hipotonía muscular, el estado de los reflejos osteotendinosos y presencia de otros elementos como fasciculaciones de la lengua o rigidez articular. Resulta importante resaltar que no todos los casos que se registraron reportaban los estudios complementarios completos tales como: electromiografías, CPK, biopsias de músculo y un seguimiento clínico más minucioso.

Fueron clasificados los tipos de AME (I a III), de acuerdo a la edad de debut de los síntomas en: tipo I, debut antes de los 6 meses de edad; tipo II, debut entre los 6 y 18 meses de edad; tipo III, debut después de los 18 meses de edad y según los criterios diagnósticos de Munsat y Davies ${ }^{8}$.

Tabla 1. Condiciones de reacción para la amplificación de las regiones de interés en el gen SMN1 (exones 7 y 8 ) para un volumen de $\mathbf{2 5} \boldsymbol{\mu l}$

\begin{tabular}{|lc|}
\hline Reactivos & Concentración \\
\hline Buffer STR* & $0,2 \mathrm{mM}$ \\
Oligonucleótido 7A & $0,8 \mathrm{mM}$ \\
(5' AGA CTA TCA ACT TAA TTT CTG ATC A 3') & \\
Oligonucleótido 7X & \\
(5' CCT TCC TTC TTT TTG ATT TTG TTT 3') & \\
$\begin{array}{l}\text { Oligonucleótido 8A } \\
\text { (5' TAT GTA ATA ACC AAA TGC AAT 3') }\end{array}$ & $0,8 \mathrm{mM}$ \\
Oligonucleótido 8C & \\
(5' ATA AAC TAC AAC ACC CTT CTC A 3') & \\
Enzima Taq (5 U/ml) & $0,2 \mathrm{ml}$ \\
\hline
\end{tabular}

*Este buffer contiene una mezcla de los desoxinucleótidos trifosfatos de Adenina, Timina, Citosina y Guanina.
Se calculó la frecuencia de AME por 100.000 habitantes para cada provincia del país, de acuerdo al rango de edad de la población estudiada ( $<1$ año hasta 45 años) y los datos demográficos del Anuario Estadístico de Salud 2010.

\section{Caracterización de las deleciones}

El estudio de las deleciones en el gen SMN1 se llevó a cabo mediante la técnica de la reacción en cadena de la polimerasa (PCR) con la enzima Taq Polimerasa Promega $5 \mathrm{U} / \mathrm{ml}$ que amplificó las regiones del gen SMN en un termociclador modelo Minicicle MJ Research, Inc., v, 2.0 y cebadores correspondientes a los exones $7 \mathrm{y}$ 8 del gen, y las condiciones de reacción descritas en la tabla 1, estandarizadas en el Laboratorio.

Los productos amplificados de los exones 7 y 8 fueron digeridos con enzimas DraI y DdeI Sigma respectivamente y analizados en gel de agarosa al 2\% según la técnica descrita por Van der Steege et al y Parsons et $\mathrm{al}^{10,11}$.

\section{Resultados}

\section{Clasificación de los tipos de AME}

En cuanto a los tipos de AME 62 pacientes fueron diagnosticados como AME I (59\%), 30 pacientes como AME II $(28,6 \%)$ y 13 pacientes con AME III $(12,4 \%)$ (figura $1)$.

Estos resultados coinciden con lo planteado por otros autores, en estudios con grandes series de casos y donde se reportan una mayor frecuencia de AME tipo $\mathrm{I}^{12-14}$.

\section{Caracterización molecular}

De los 105 pacientes estudiados con sospecha de AME, 38 (36,2\%) resultaron tener deleción de los exones 7 y 8 del gen SMN, $37(35,2 \%)$ tuvieron solamente deleción del exón 7 de gen SMN y 30 $(28,6 \%)$ no presentaron deleción de los exones 7 ni 8 del gen SMN (figura 2).

Se plantea que entre el $95-98 \%$ de los pacientes con AME poseen deleciones de los exones 7 y 8 , en la copia telomérica del 


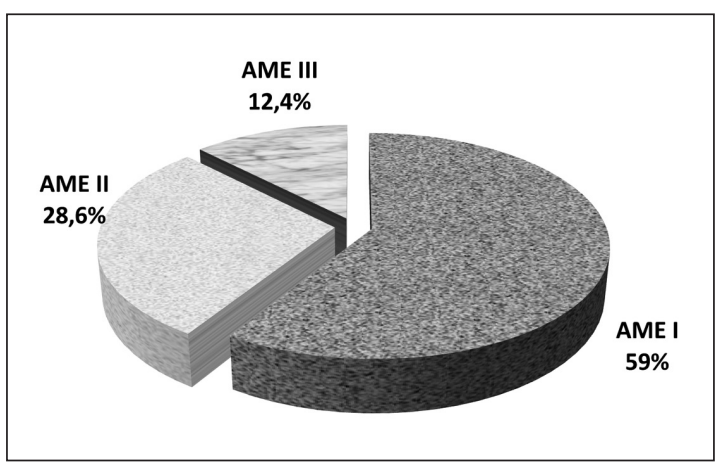

Figura 1. Tipos y porcientos de AMEi en el presente estudio.

gen $\mathrm{SMN} 1^{15}$, sin embargo, en la serie de casos presentada se obtuvo una positividad inferior $(71,4 \%)$.

En la figura 3 se muestra la frecuencia de AME por 100.000 habitantes por provincias de todo el país, con confirmación molecular (Gen SMN) y registrados en nuestro departamento en período 1997-2010.

Las frecuencias más altas se encuentran en la región occidental de país 64,2\% (70/109) (Pinar del Río, La Habana, Artemisa, Mayabeque, Matanzas e Isla de la Juventud). Las frecuencia más bajas las encontramos en el área oriental de país, donde hay ciudades con el segundo y tercer lugar en la densidad de población de la isla 22\% (24/109) Camaguey, Las Tunas, Holguín, Granma, Santiago de Cuba y Guantánamo.

\section{Discusión}

Se presentan los resultados de la caracteri-

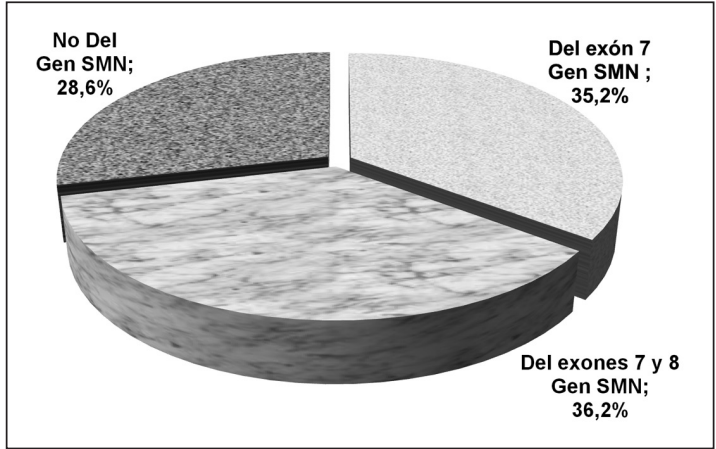

Figura 2. Caracterización molecular de la serie de casos en el presente estudio.

zación molecular y geográfica de los pacientes con AME de la Isla de Cuba.

Los resultados de la caracterización molecular pudieran estar relacionados con las características de este estudio que abarca un período de tiempo extenso, donde en un inicio predominó la inexperiencia en los especialistas y/o imprecisiones en el diagnóstico clínico y la falta de estudios complementarios necesarios como la electromiografía. En un inicio se recibieron muestras de pacientes de todas las provincias del país que fueron remitidas por médicos de diferentes especialidades, por lo que los criterios clínicos no eran uniformes.

Con el tiempo los estudios clínicos se han ido perfeccionando. La introducción de otros estudios complementarios junto a la confirmación molecular de la AME en el país y el trabajo de un equipo multidisciplinario en nuestro instituto que reevalúa y sigue todos los casos remitidos desde otros servicios del país, ha contribuido a una mayor positividad en los

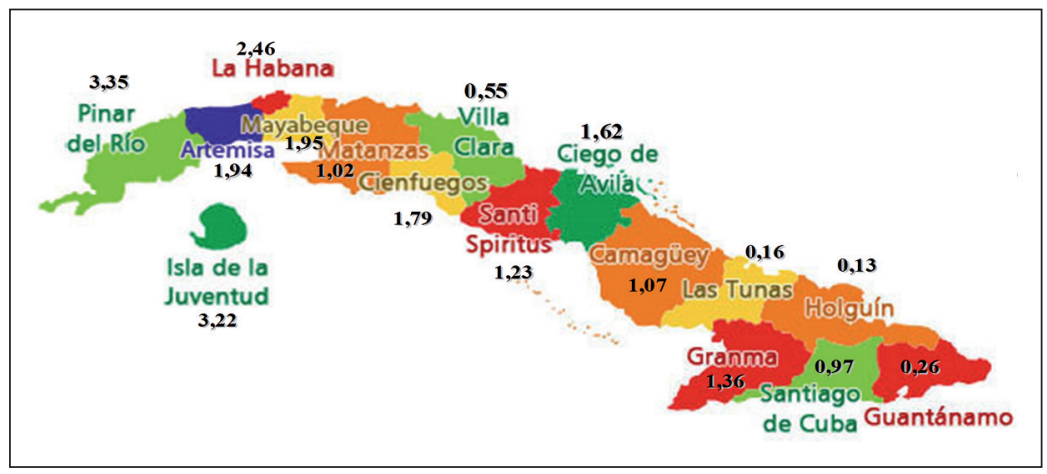

Figura 3. Frecuencias de AME por 100.000 habitantes, en cada provincia del país, confirmados molecularmente en la serie de casos registrados por el Departamento de Neurogenética del INN de La Habana, en el período 1997-2010. 
hallazgos moleculares del gen SMN en las últimas etapas del presente estudio.

La frecuencia de deleción de los exones 7 y 8 en nuestro estudio resultó diferente a la encontrada en pacientes negros sudafricanos. Ellos reportaron un 47\% (9/19) de pacientes con deleción homocigótica de la copia telomérica del exón 7 , pero no del exón 8 . Por otra parte, deleciones homocigóticas de los exones 7 y 8 se han reportado en el $85 \%$ de pacientes con AME españoles y en el $84,6 \%$ de pacientes chilenos $^{16-18}$.

Nuestros resultados evidencian la contribución de genes SMN de origen caucásico y negroides a la población cubana, que con un alto índice de mestizaje, se ha reportado una menor incidencia de estas enfermedades en la población cubana en relación a otros estudios en poblaciones caucásicas europeas ${ }^{7,18-20}$.

Los resultados de la distribución geográfica son coincidentes con una frecuencia más alta de mestizos y de población de origen étnico negroide en el este del país y corroboran la baja incidencia en Cuba detectada por Zaldívar et al, 2005, que reportaron bajas frecuencias de AME I en pacientes con los antepasados africanos $^{7}$.

Baste decir, que en la formación de la nacionalidad cubana, en siglos anteriores intervinieron asentamientos de grandes poblaciones negras africanas traídos como esclavos a trabajar en las mayores plantaciones de caña de azúcar y café del Caribe, ubicadas en la región oriental del país ${ }^{21}$.

Contrariamente una frecuencia relativamente alta se observa en las provincias occidentales donde existen densidades de población de origen Caucásico más altas y donde hubo plantaciones de tabaco y cultivos varios en cuya mano de obra fue menor la presencia de esclavos africanos y mayor la presencia de otras poblaciones caucásicas como Canarios, y de otras regiones de España.

Desde mediados del siglo XIX, se plantea, irrumpió también en estas plantaciones, mano de obra de origen asiático, por lo que la población cubana actual es el resultado del gran mestizaje de líneas ancestrales Caucásicas y Negroides principalmente y que ha sido reportado en la literatura ${ }^{22,23}$.
Estudios similares en la anemia drepanocítica y la esclerosis lateral amiotrófica también han informado una distribución diferencial de estas enfermedades según la diversidad de ancestros de la población cubana ${ }^{23,24}$.

Estos resultados constituyen el primer reporte en la población caribeña, donde no abundan estudios, al menos publicados, sobre enfermedades neurodegenerativas.

\section{Referencias}

1.- Wirth B: An update of the mutation spectrum of the survival motor neuron gene (SMN1) in autosomal recessive spinal muscular atrophy (SMA). Hum Mutat 2000; 15: 228-37.

2.- Brahe C, Bertini E: Spinal muscular atrophies: recent insights and impact on molecular diagnosis. J Mol Med 1996; Oct; 74 (10): 555-62.

3.- Markowitz JA, Priyamvada S, Darras BT: Spinal Muscular Athrophy: A Clinical and Research update. Pediatric Neurology 2012; 461-2.

4.- Pearn J: Incidence, relevance and gene frequency studies of chronic spinal muscular atrophy. J Med Genet 1978; 15: 409-13.

5.- Mostacciulo ML, Danieli GA, Trevisan C, Muller E, Angelini $C$ : Epidemiology of spinal muscular atrophies in a sample of the Italian population. Neuroepidemiology 1992; 11: 34-8.

6.- Thieme A, Mitulla B, Friedemann S, Spiegler AW: Epidemiological data on Werdnig-Hoffmann disease in Germany (West-Thuringen). Human Genet 1993; 91: 295-97.

7.- Zaldivar T, Montejo Y, Acevedo AM, et al: Evidence of reduced frequency of spinal muscular atrophy type I in the Cuban population. Neurology 2005; 65: 636-8.

8.- Munsat TL, Davies KE: International SMA consortium meeting. Germany). Neuromuscul Disord 1992; 2 (5-6): 423-8.

9.- Anuario Estadístico de Salud 2010: Dirección Nacional de Registros Médicos y Estadísticas de Salud. Disponible en: http://www.sld.cu/servicios /estadisticas/. [15.5.2012].

10.- van der Steege G, Grootscholten PM, van der Draaijers $T G$, et al: PCR-based DNA test to confirm clinical diagnosis of autosomal recessive spinal muscular atrophy. Lancet 1995; 345 (8955): 985-6.

11.- Parsons DW, McAndrew PE, Iannaccone ST, Mendell $J R$, Burghes AH, Prior TW: Intragenic telSMN muta- 
tions: frequency, distribution, evidence of a founder effect, and modification of the spinal muscular atrophy phenotype by cenSMN copy number. Am J Hum Genet 1998; 63 (6): 1712-23.

12.- Ludvigsson P, Olafsson E, Hauser WA: Spinal muscular atrophy. Neuroepidemiology 1999; 18: 265-9.

13.- Munsat TL, Davies KE: International SMA consortium meeting. (26-28) June 1992, Bonn, Germany). Neuromuscul Disord 1992; 2 (5-6): 423-8.

14.- Bussaglia E, Clermont $O$, Tizzano E, et al: A frame-shift deletion in the survival motor neuron gene in Spanish spinal muscular atrophy patients. Nat Genet 1995; 11 : 335-7.

15.- Markowitz JA, Priyamvada S, Darras BT: Spinal Muscular Athrophy: a clinical and research update. Pediatric Neurology 2012; 46: 1-12.

16.- Stevens $G$, Yawitch T, Rodda J, Verhaart S, Krause A: Different molecular basis for spinal muscular atrophy in South African black patients. Am J Med Genet 1999 Oct 29; 86 (5): 420-6.

17.- Castiglioni C, Levicán J, Rodillo E, et al: Atrofia muscular espinal: Caracterización clínica, electrofisiológica y molecular de 26 pacientes. Rev Med Chile 2011; 139: 197-204.
18.- Cusco I, Barcelo MJ, del Río E, et al: Characterisation of SMN hybrid genes in Spanish patients: de novo, homozygous y and compound heterozygous cases. Hum Genet 2001; 108 (3): 222-9.

19.- Vaidla E, Talvik I, Kulla A, et al: Descriptive Epidemiology of Spinal Muscular Atrophy Type I in Estonia. Neuroepidemiology 2006; 27: 164-8.

20.- Mazzei R, Gambardella A, Conforti FL, et al: Gene conversion events in adult-onset spinal muscular atrophy. Acta Neurol Scand 2004; 109: 151-4.

21.- Martínez-Fuentes AJ: El status del concepto de raza en la Antropología biológica contemporánea. Documento de Cuba Arqueológica. http://www.cubaarqueologica. org. [21.4.2012].

22.- Cintado A, Companioni AO, Nazabal M, et al: Admixture estimates for the population of Havana City. Annals of Human Biology 2009; 36 (3): 350-60.

23.- Granda H, Gispert S, Dorticos A, et al: Cuban programme for prevention of sicklecell disease. Lancet 1991; 337: 152-53.

24.- Zaldivar T, Gutiérrez J, Lara G, Carbonara M, Logroscino $G$, Hardiman $O$ : Reduced frequency of ALS in an ethnically mixed population: A population-based mortality study. Neurology 2009; 72: 1640-5. 